\title{
The effect of feed-based vaccination on tilapia farm endemic for streptococcosis
}

\begin{abstract}
A tilapia farm experiencing endemic streptococcosis was selected to study the effect of vaccination with a feed-based vaccine on naturally ocurring streptococcosis. A total of 9000 red tilapia, Oreochromis niloticus $\times$ Oreochromis mossambicus of $100 \pm 20 \mathrm{~g}$ were divided into 9 cages. Fish of Group 1 in cages 1, 2 and 3 were not vaccinated. Group 2 in cages 4, 5 and 6 were vaccinated on days 0 and 14 (single booster) while Group 3 in cages 7, 8 and 9 were vaccinated on days 0,14 and 42 (double booster). Vaccination was done by oral administration of the feed-based bacterin vaccine at $4 \%$ bodyweight. Samples of serum for antibody study and the brain, eyes and kidney for bacterial isolation were collected at 14-day intervals. The study was carried out during the critical months between April and June. Following vaccination and booster, there was significant $(\mathrm{p}<0.05)$ increase in the antibody levels in all vaccinated groups from week 1 that reached the peak at week 3 before declining gradually until week 6 . However, second booster on week 6 significantly $(p<0.05)$ increased the antibody level that remained high until the end of the 16-week study period (double booster). Streptococcus agalactiae was isolated at the start of the experiment (day 0) at an average of $10 \pm 5.0 \%$ of the sampled fish. In week 4 , the isolation rate was $13 \pm 5.7 \%$ but increased to $18 \pm 7.6 \%$ in week 8 , to $25 \pm 10.0 \%$ in week 10 , to $28 \pm 5.8 \%$ in week 12 and 25 $\pm 7.3 \%$ in week 14 . The average isolation rate was $28 \pm 7.2 \%, 18 \pm 7.1 \%$ and $13 \pm 8.2 \%$ of the fish sampled from unvaccinated, single booster and double booster groups, respectively. At the end of the study period, the survival rate was $45.2 \pm 2.45 \%$ for unvaccinated, $65.3 \pm$ $4.8 \%$ for single booster and $75.1 \pm 2.1 \%$ for double booster groups. Vaccinating fish in endemic farm might not eliminate the disease but was able to significantly improve the survival rate.
\end{abstract}

Keyword: Efficacy; Feed-based vaccine; Streptococcosis; Endemic farm 